\title{
Photoproduction of electron-positron pairs in bent single crystals
}

\author{
Yu. A. Chesnokov and V. A. Maisheev \\ Institute for High Energy Physics, 142281, Protvino, Russia \\ D. Bolognini, S. Hasan, and M. Prest \\ Università dell'Insubria and INFN Milano Bicocca, Como, Italy \\ E. Vallazza \\ INFN Trieste, Padriciano 99, Trieste, Italy \\ (Received 16 March 2010; published 29 July 2010)
}

\begin{abstract}
The process of photoproduction of electron-positron pairs in bent single crystals is considered. This consideration is based on the theory of coherent pairproduction in straight single crystals and take into account the linear polarization of the photon beam. In particular, it is shown that the probability of the process for $\gamma$ quanta with energies from $100 \mathrm{GeV}$ is significantly higher than the one in an amorphous medium. The positive features of the photoproduction in bent crystals compared to straight ones are underlined from the point of view of possible applications.
\end{abstract}

DOI: 10.1103/PhysRevSTAB.13.070706

PACS numbers: $61.80 . \mathrm{Cb}, 29.27 .-\mathrm{a}, 29.30 .-\mathrm{h}$

\section{INTRODUCTION}

In recent years several experiments studying different processes of charged particle interaction with bent single crystals were performed [1-3]. In particular, the process of radiation emission of ultrarelativistic electrons and positrons moving in the planar fields of a bent single crystal was investigated. The experiments have shown a significant increase of the radiation energy losses of electrons and positrons in conditions of volume reflection [2,3]. The present paper considers the process of $e^{ \pm}$-pair creation by high energy photons moving in a bent single crystal with a constant curvature. This study is based on the theory of coherent $e^{ \pm}$pair production [4,5] in straight single crystals. The photoproduction of $e^{ \pm}$pairs in a periodically deformed single crystal was studied in [6]. This study and the consequent results depend essentially on the periodic properties of the process and, hence, cannot be used directly for the case of a bent single crystal with a constant curvature.

Sections II and III are devoted to the mathematical description of the motion of charged and neutral particles in bent crystals and of the photoproduction process while Sec. IV describes the application of this model to two possible photon energies (120 and $1000 \mathrm{GeV}$ ).

\section{MOTION OF CHARGED AND NEUTRAL PARTICLES IN THE BENT PLANAR FIELDS OF A SINGLE CRYSTAL}

The motion of ultrarelativistic charged particles in bent single crystals can be described with the help of the following equations [7]:

$$
\begin{gathered}
E_{0} \beta^{2} v_{r}^{2} /\left(2 c^{2}\right)+U(r)+E_{0} \beta^{2}(R-r) / r=E=\mathrm{const} \\
d y / d t=v_{y}=\mathrm{const} \\
v_{z}=r d \phi / d t \approx c\left(1-\frac{1}{2 \gamma^{2}}-\frac{\left(v_{r}^{2}+v_{y}^{2}\right)}{2 c^{2}}\right)
\end{gathered}
$$

These equations are valid in the cylindrical coordinate system $(r, \phi, y) . v_{r}$ is the component of the particle velocity in the radial direction, $v_{y}$ the one along the $y$ axis, and $v_{z}$ the tangential one; $R$ is the bending radius of the single crystal, $E_{0}$ and $\gamma$ are the particle energy and its Lorentz factor, $E$ is the constant value of the radial energy, $U(r)$ is the crystal one dimensional potential, $c$ the velocity of light, and $\beta$ the ratio of the particle velocity to the velocity of light. This paper considers the planar case where the scattering is due to the interaction of the particles with the set of crystallographic planes located normally to the $(r, \phi)$ plane. In practice it means that $v_{y} / c \gg \theta_{a c}$ but $v_{y} / c \ll 1$ for ultrarelativistic particles, where $\theta_{a c}$ is the critical angle of axial channeling.

Equation (1) can be transformed in the following way:

$$
E_{0} \beta^{2} v_{x}^{2} /\left(2 c^{2}\right)+U(x)+E_{0} \beta^{2} x / R=E,
$$

where $x$ is the local Cartesian coordinate which is related to the cylindrical coordinate $r$ through the expression $x=$ $R-r$ and $v_{x}=v_{r}$, and the $r$ value in the denominator of Eq. (1) has been changed to $R$. This change introduces a negligible error in the experimental measurement (of the 


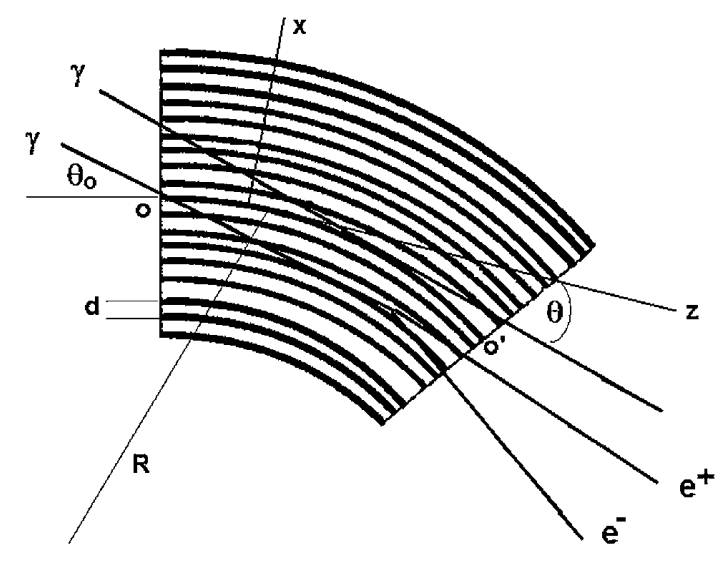

FIG. 1. Production of $e^{ \pm}$pairs by a high energy photon moving in a bent single crystal. ( $x y z)$ is the local Cartesian coordinate system. The $y$ axis is directed perpendicularly to the plane of the figure; $d$ is the interplanar distance, $\theta_{0}$ is the entrance angle, and $\theta$ is the variable angle relative to the (110) plane of the single crystal.

order of $x / R$ ). Finally, $E$ is the transversal energy. Figure 1 illustrates the geometry of the $e^{ \pm}$-pair production process and the local coordinate system which will be used in the following considerations. For neutral particles $(\beta=1$ for photons) Eq. (4) should be substituted with

$$
\frac{E_{\gamma} v_{x}^{2}}{2 c^{2}}+\frac{E_{\gamma} x}{R}=E
$$

where $E_{\gamma}$ is the photon energy. This leads to

$$
\begin{aligned}
& x=x_{0}+v_{o} t-\frac{t^{2} c^{2}}{2 R}, \\
& \theta=\frac{1}{c} \frac{d x}{d t}=\theta_{0}-\frac{c t}{R},
\end{aligned}
$$

where $x_{0}$ and $v_{o}$ are the initial coordinate and velocity of the photon in the local Cartesian coordinate system $[t=0$ corresponds to the $x_{0}$ coordinate and $v_{o}=c \theta_{0}$, and hence $\left.E=E_{\gamma} v_{0}^{2} /\left(2 c^{2}\right)+E_{\gamma} x_{0} / R\right]$. Equation (7) describes the variation of the direction of the photon motion relative to the planar electric field (in the local coordinate system) and plays an important role in the description of the process.

The process of photoproduction in a bent single crystal is very similar to the process of radiation emission of electrons and positrons undergoing volume reflection in such a crystal. The photoproduction probability depends on the character of the electron (positron) motion. However, differently from radiation emission, photoproduction is a threshold process, which introduces some peculiarities. The motion of charged particles was investigated in [7] and those results will be used in the following.

\section{PROBABILITY OF PHOTOPRODUCTION OF $e^{ \pm}$PAIRS IN THE BENT PLANES OF A SINGLE CRYSTAL}

Let us consider the motion of a high energy photon which forms an initial entrance angle $\theta_{0}$ with the crystal and moves towards the tangency point. During its motion, the photon intersects the bent planes of the crystal (see Fig. 1). In the local Cartesian coordinates, this motion is described by Eq. (6). At a time $t_{1}$ the photon has the corresponding coordinate $x_{0}<x<x_{c}$, where $x_{c}$ is the coordinate of the tangency point. If it is assumed that the bending radius of the single crystal is large enough, the influence of the curvature on the process in some area $x_{0}<$ $x_{1}<x<x_{2}<x_{c}$ can be neglected allowing one to use the theory of coherent pair production in straight crystals for the description of the process in the area $x_{1}, x_{2}$. The theory of coherent pair production in crystals requires the following conditions to be respected [4,5]: (i) the mean angle of particle deflection from the straight line on the formation length $l_{f}$ should be $\ll 1 / \gamma_{f}$, where $\gamma_{f}=E_{\gamma} / m c^{2}$ ( $m$ is the mass of the electron); (ii) the differences of the periods for neighboring crossings should be small; (iii) the characteristic parameter $\rho$ should be $\ll 1$. The first condition in the case under consideration means that $l_{f} / R \ll 1 / \gamma_{f}$. The formation length for the process may be written as

$$
l_{f}^{-1}=\frac{m c^{2}}{2 E_{\gamma} \lambda_{c} x_{e}\left(1-x_{e}\right)} \geq \frac{2 m c^{2}}{E_{\gamma} \lambda_{c}}
$$

where $x_{e}$ is the relation of the electron (or positron) energy to the photon energy and $\lambda_{c}$ is the Compton wavelength of the electron. It follows that

$$
R \gg \gamma_{f}^{2} \lambda_{c} / 2 .
$$

The second condition can be explained in the following way. In a straight single crystal the coherent process of pair production takes place because of the periodical perturbation of the photon state at the crossing of the atomic planes. The same explanation can be also applied to a bent crystal in the limited area $x_{1}, x_{2}$. Finding $t$ as a function of $x$ [see Eq. (6)] and taking into account that the atomic planes have a period equal to $d$, it is possible to calculate the time periods of the planes crossing during the photon motion. The total number of periods (from the point of entrance to the tangency point) is $\approx \theta^{2} R /(2 d)$. With the help of Eqs. (1)-(4), one can compute the parameter $\rho=$ $2 \gamma^{2}\left\langle\left(V_{x}-\left\langle V_{x}\right\rangle\right)^{2}\right\rangle / c^{2}$ for positrons or electrons. Thus the following approximate relation can be written

$$
\left\langle\left(V_{x}-\left\langle V_{x}\right\rangle\right)^{2}\right\rangle \approx \frac{c^{2}}{8 E_{0} \beta^{2}} \frac{\left[\left\langle U^{2}(x)\right\rangle-\langle U(x)\rangle^{2}\right]}{\left(E-E_{0} \beta^{2} x / R\right)},
$$

where $\langle U(x)\rangle$ and $\left\langle U^{2}(x)\right\rangle$ are the mean and mean square of the planar potential and $E \approx E_{0} \theta_{c}^{2} / 2+U\left(x_{1}\right)$, where the $\theta_{c}$ angle corresponds to the $x_{1}$ coordinate of the point of the particle creation. This relation is violated in the region 
close to the tangency point; in this region in fact a more exact calculation gives an approximately twice larger result. With the condition $E_{0} \beta^{2} x / R \ll E$, it follows that

$$
\rho_{e} \approx \frac{\left[\left\langle U^{2}(x)\right\rangle-\langle U(x)\rangle^{2}\right]}{2 m^{2} c^{4} \theta_{c}^{2}} .
$$

Note that $\left\langle U^{2}(x)\right\rangle-\langle U(x)\rangle^{2}=42.3 \mathrm{eV}^{2}$ for the silicon (110) plane.

It should be noted that, for coherent pair production, the following limit on the angle holds:

$$
\theta_{l}(n) \geq 2 m c^{2} /\left(E_{\gamma} \lambda_{c} G n\right),
$$

where $n$ is a positive integer number and $G=2 \pi / d$. $n$ is the number of the harmonics of the potential. It is well known that the main contribution to the process is given by the first harmonic, while the contribution of the fourth and higher order harmonics is small. Thus, Eq. (12) shows that, for small enough angles, the coherent process of pair production is absent.

At this point, the conditions for coherent pair production in the (110) bent silicon planes can be investigated. The analysis will be performed for two photon energies equal to 120 and $1000 \mathrm{GeV}$. From Eq. (9) it follows that the bending radius has to be $R \gg 0.8$ and $80 \mathrm{~cm}$, respectively. With the help of Eq. (12) it is found that $\theta_{l}(1)=0.4$ and $0.04 \mathrm{mrad}$, respectively, while the $\rho_{e}$ parameter should be $\rho_{e} \leq 1$ at $\theta_{c} \approx 0.01 \mathrm{mrad}$. The ratio of the neighboring periods for $R=250 \mathrm{~cm}$ at $\theta \approx 0.05 \mathrm{mrad}$ differs from $1 \%$ to $3 \%$, while for $R=1000 \mathrm{~cm}$ it differs of $1 \%$. Increasing the $\theta$ angle, this value becomes significantly better.

It is evident that also in bent crystals the conditions for coherent pair production can be met in small areas:

$$
d \mathcal{W}\left(L, \theta_{0}\right)=W[\theta(t)] c d t,
$$

where $\mathcal{W}\left(L, \theta_{0}\right)$ is the total probability of pair production on the full length $L$ of a single crystal by a photon with an entrance angle $\theta_{0}$ and $W(\theta)$ is the probability of pair production per unit length by a photon moving with an angle $\theta$. Taking into account Eqs. (6) and (7) it is found that

$$
\mathcal{W}\left(L, \theta_{0}\right)=R\left|\int_{\theta_{1}}^{\theta_{0}} W(\theta) d \theta\right|,
$$

where $\theta_{1}=\theta_{0}-L / R$ in accordance with Eq. (7) and the $W$ functions are equal to (see for example $[4,5]$ )

$$
\begin{aligned}
W_{0}(\theta) & =\frac{W_{\|}+W_{\perp}}{2} \\
& =\frac{n B \sigma_{0} E_{\gamma} \lambda_{c}}{4 m c^{2}} \sum_{i=1}^{\infty} \Phi(G i)(G i)^{2} F_{2}\left(z_{i}\right)+n \sigma_{A}, \\
D(\theta) & =\frac{W_{\|}-W_{\perp}}{2}=\frac{n B \sigma_{0} E_{\gamma} \lambda_{c}}{16 m c^{2}} \sum_{i=1}^{\infty} \Phi(G i)(G i)^{2} F_{1}\left(z_{i}\right),
\end{aligned}
$$

where $\sigma_{A}$ is the cross section of pair production in a nonoriented crystal [4], $\sigma_{0}=\alpha_{e} Z^{2} r_{e}^{2}, \alpha_{e}$ is the fine-structure constant, $Z$ is the atomic number of the crystal material, $r_{e}$ is the classical electron radius, $B=16 \pi^{2} /\left(N_{S} \Delta\right), N_{S}$ is the number of atoms per elementary cell (with a volume $\Delta$ ) of a crystal, $n=N_{S} / \Delta$ is the atomic density, $G=2 \pi / d$, and $d$ is the interplanar distance. The variable $z_{i}$ is defined from the relation

$$
z_{i}=\frac{2 m c^{2}}{E_{\gamma} \lambda_{c} G i|\theta|}
$$

Other definitions are

$$
\Phi(G i)=|S(G i)|^{2}[1-F(G i)]^{2} e^{-A(G i)^{2}} /(G i)^{4},
$$

where $S(G i)$ is the structure factor of the reciprocal vector $G i, F$ is the atomic form factor, and $A$ is the mean square of the thermal atomic vibrations. The functions $F_{1}, F_{2}$ are the following:

$$
\begin{gathered}
F_{1}(z)=z^{4}\left(\ln \frac{1+\sqrt{1-z}}{1-\sqrt{1-z}}+\frac{2 \sqrt{1-z}}{z}\right) \eta(z) \eta(1-z), \\
F_{2}(z)=z^{2}\left(\left(1+z-z^{2} / 2\right) \ln \frac{1+\sqrt{1-z}}{1-\sqrt{1-z}}\right. \\
-\sqrt{1-z}(1+z)) \eta(z) \eta(1-z)
\end{gathered}
$$

where the $\eta(z)$ function is equal to 1 or 0 at $z \geq 0$ and $z<0$, respectively.

The equations presented here reflect the fact that the crystal can feel the linear polarization of the photons and hence $W_{\|}$and $W_{\perp}$ are the probabilities of pair production per unit length for photons with a linear polarization along a planar electric field and along a perpendicular plane, respectively. Substituting these probabilities in Eq. (14), one can obtain the total probabilities in a bent single crystal for the corresponding photon polarization states. It is clear that the $W_{0}$ probability corresponds to an unpolarized beam. After the integration [in accordance with Eq. (14)], one has

$$
\begin{aligned}
\mathcal{D}_{0}= & \frac{\mathcal{W}_{\|}-\mathcal{W}_{\perp}}{2} \\
= & \frac{n B \sigma_{0} R}{8} \sum_{i=1}^{\infty} \Phi(G i) G i\left|\mathcal{F}_{1}\left[z_{i}\left(\theta_{0}\right)\right]-\mathcal{F}_{1}\left[z_{i}\left(\theta_{1}\right)\right]\right| \\
\mathcal{W}_{0}= & \frac{\mathcal{W}_{\|}+\mathcal{W}_{\perp}}{2} \\
= & \frac{n B \sigma_{0} R}{2} \sum_{i=1}^{\infty} \Phi(G i) G i \mid \mathcal{F}_{2}\left[z_{i}\left(\theta_{0}\right)\right] \\
& \quad-\mathcal{F}_{2}\left[z_{i}\left(\theta_{1}\right)\right]|+R| \theta_{0}-\theta_{1} \mid n \sigma_{A}
\end{aligned}
$$


where

$$
\begin{aligned}
\mathcal{F}_{1}(z)= & -\frac{2}{3} \sqrt{1-z}-\frac{(36 z+4)}{45}(1-z)^{3 / 2} \\
& -\frac{2}{15}(1-z)^{5 / 2}+\frac{z^{3}}{3} \ln \frac{1+\sqrt{1-z}}{1-\sqrt{1-z}}, \\
\mathcal{F}_{2}(z)=- & \frac{8}{3} \sqrt{1-z}+\left(\frac{47}{45}+\frac{2}{5} z\right)(1-z)^{3 / 2} \\
+ & \frac{1}{15}(1-z)^{5 / 2}+\left(z+\frac{z^{2}}{2}-\frac{z^{3}}{6}\right) \ln \frac{1+\sqrt{1-z}}{1-\sqrt{1-z}} .
\end{aligned}
$$

The variable $z_{i}$ depends on the entrance angle $\theta_{0}$ and on the final angle $\theta_{1}$ accordingly to Eq. (7). Besides, the functions $\mathcal{F}_{1}, \mathcal{F}_{2}$ are $=0$ when $|z|>1$. These relations are valid for the case where $\theta_{0}$ and $\theta_{1}$ have the same sign. In the case where these values have a different sign, the correct result
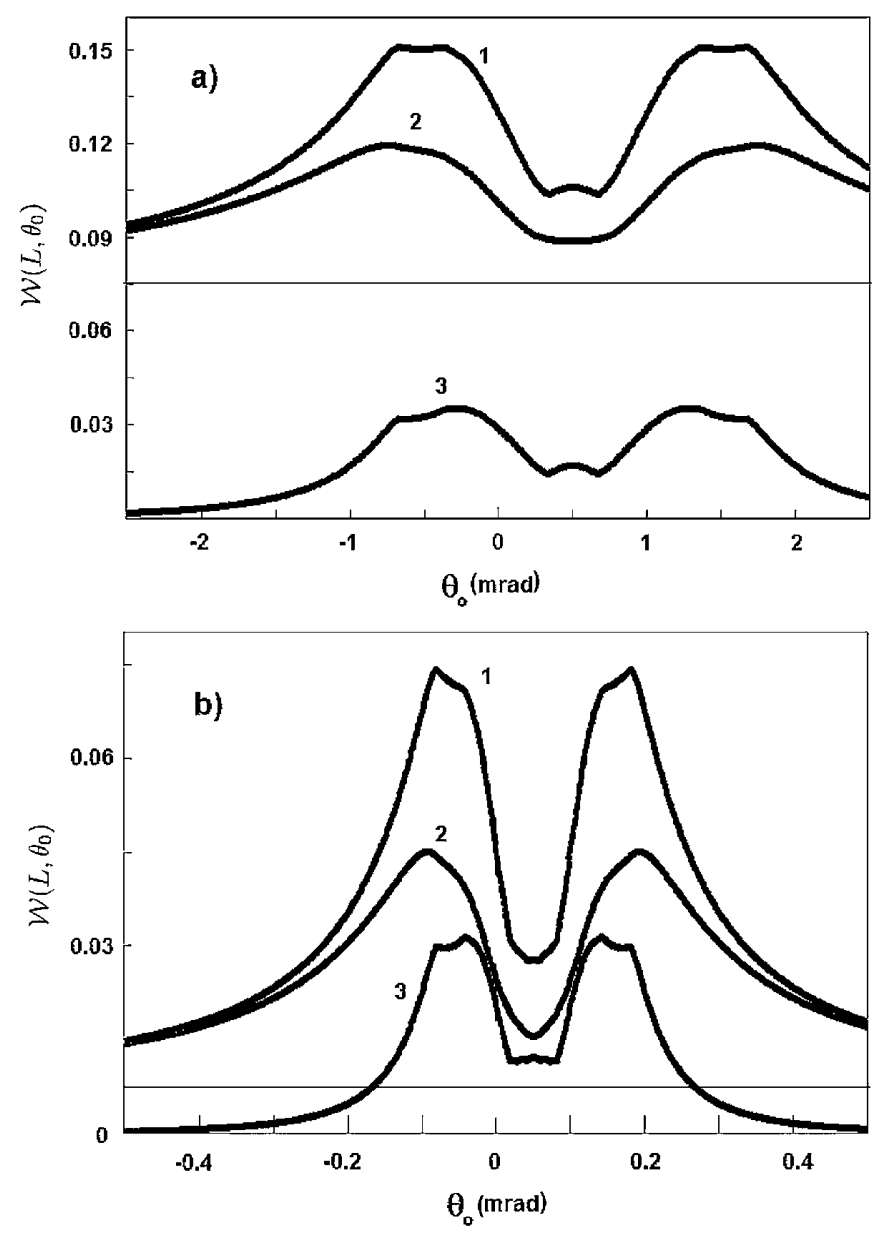

FIG. 2. Production of $e^{ \pm}$pairs by a high energy photon moving in a bent single crystal. ( $x y z)$ is the local Cartesian coordinate system. The $y$ axis is directed perpendicularly to the plane of the figure; $d$ is the interplanar distance, $\theta_{0}$ is the entrance angle, and $\theta$ is the variable angle relative to the (110) plane of the single crystal. is the sum of the two values, with the first value equal to the probability from $\theta_{0}$ to 0 and the second value equal to the probability from 0 to $\theta_{1}$.

It should be noted that this description is valid for a thin enough crystal where $W_{\|}, W_{\perp} \ll 1$. For a thicker crystal, a more complicated theory should be used (see for example [8]) which takes into account the changing of the polarization states during the propagation of the photons. The corresponding differential probabilities can be found in the literature $[4,5]$.

\section{EXAMPLES OF CALCULATION}

The method has been applied to compute the expected results with two different photon energies: 120 and $1000 \mathrm{GeV}$. The first value has been chosen given the experimental possibility offered by the INSURAD test setup on the CERN SPS H4 beam line, while the second value represents approximately the limit of applicability of the method. Figure 2 illustrates the behavior of the total
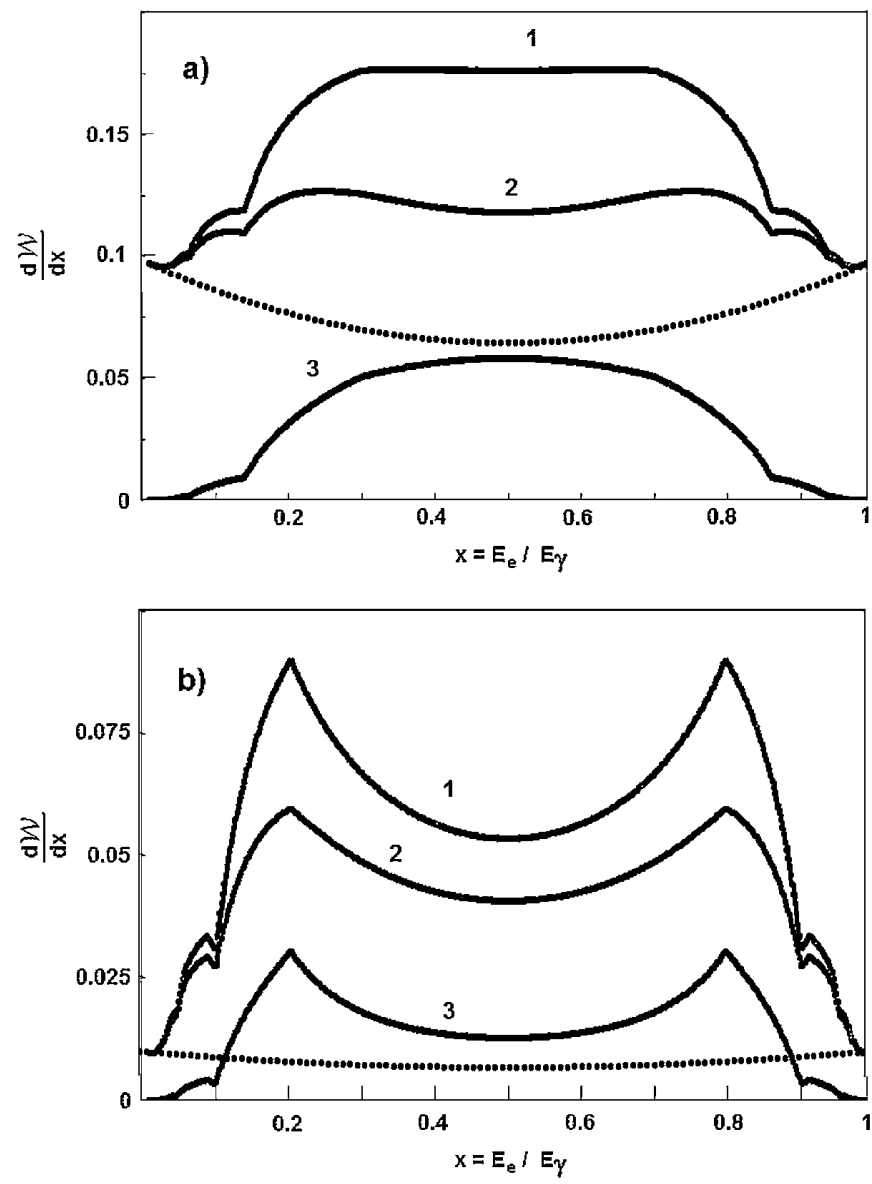

FIG. 3. Spectral distributions of the electrons (positrons) produced in a single bent silicon crystal at the conditions of Fig. 2, for the two cases (a) and (b). The entrance angle is $\theta_{0}=$ $+1.5 \mathrm{mrad}$ (a) and $+0.2 \mathrm{mrad}$ (b). The numbers near the curves indicate the probabilities as in Fig. 2. The black point curves are the ones for nonoriented single crystals. 
probability of pair production as a function of the entrance angle $\theta_{0}$. One can see the nonsymmetrical curves with respect to the entrance angle. At both energies the region of orientation where the difference $\mathcal{W}_{\|}-\mathcal{W}_{\perp}$ is a measurable quantity is evident.

The differential spectra of the created positrons (electrons) are shown in Fig. 3 for two different $\theta_{0}$ entrance angles. For positive angles smaller than the angle $\theta_{0}$ corresponding to a maximum of the $d \mathcal{W}_{\|} / d x-d \mathcal{W}_{\perp} / d x$ value, the spectra have a bell-like shape [see Fig. 3(a)], while for angles larger than this angle, the typical form is presented in Fig. 3(b).

The spectra in Fig. 3 are valid for particles which were created in the bulk of the crystal (at the moment of creation). After their creation, these particles (electrons and positrons) can lose their energy due to coherent radiation processes in the electric fields of the crystal. However,
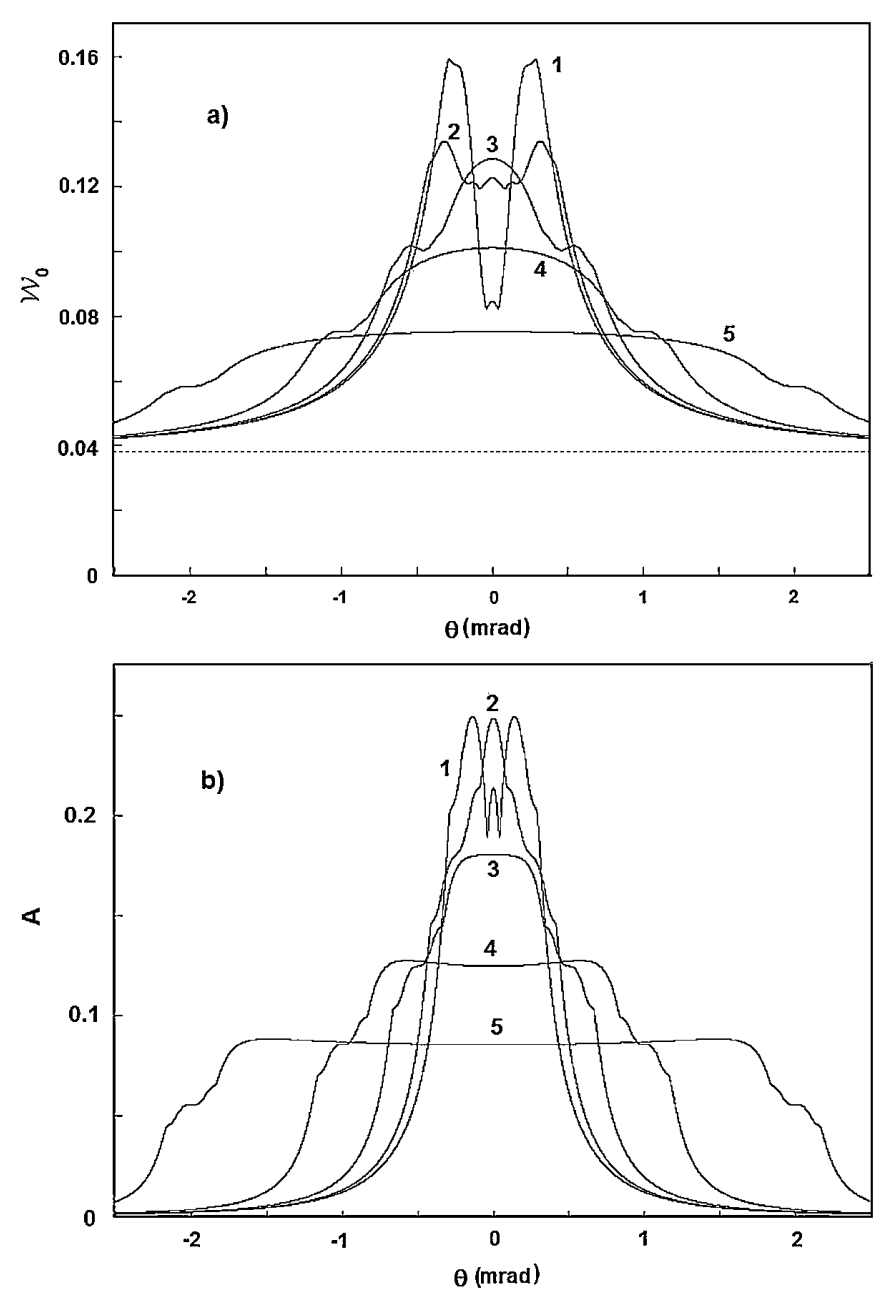

FIG. 4. The probability $\mathcal{W}_{0}$ (a) and asymmetry $A$ (b) for a $0.5 \mathrm{~cm}$ single silicon crystal at different bending radii as functions of the orientation angle $\theta$. Curves $1-5$ correspond to $R=$ $20,10,5,2.5$, and $1.25 \mathrm{~m}(\theta=0$ for a symmetric orientation of the single crystal). The point curves correspond to a nonoriented crystal. The photon energy is equal to $500 \mathrm{GeV}$. these processes are relevant only for small angles with respect to the tangency direction [9]. Hence, one can expect that the particles created at large enough $\theta_{0}$ angles have a large probability to conserve their initial energy until their exit from the crystal.

Figure 4 illustrates the behavior of the $\mathcal{W}_{0}$ probability and the $A=\mathcal{W}_{0} / \mathcal{D}_{0}$ asymmetry for different bending radii of the silicon single crystal [for a (110) planar orientation].

\section{CONCLUSIONS}

This paper shows how high energy photons moving in bent single crystals with large enough bending radii can meet the local conditions for coherent photoproduction. These conditions are violated only in a small range when the direction of the photon motion is very close to the tangent direction. According to our estimations [for a (110) silicon plane, $E_{\gamma} \leq 1000 \mathrm{GeV}$ and $R>2 \mathrm{~m}$ ], this area is about $\pm 20 \mu \mathrm{rad}$. For photon energies $\leq$ $1000 \mathrm{GeV}$, the pair production process is far from these angles. However, with the increase of the photon energy the first harmonic tends to zero and, moving towards the $\mathrm{TeV}$ range, the character of the photoproduction process will change $[10,11]$. We think that these considerations may be extended to the axial case of bent single crystals.

It should be noted that the considered pair production process in bent crystals analyzed in this paper is similar to the process of radiation emission of overbarrier positrons (electrons) experimentally investigated in $[2,3]$. The comparison with the theoretical predictions in that case was satisfactory. Theory [9] predicts correctly the range and form of the radiation energy losses spectra but with an intensity 1.7 (1.2) larger than that experimentally measured for positrons (electrons). The first calculations were performed on the basis of the quasiclassical description which was developed in [5]. Then in [12] it was demonstrated that for not large energies the theory of coherent bremsstrahlung in a local longitudinal area of the bent crystal could be used to describe radiation emission [see Eq. (13)]. A similar approach was also proposed in $[13,14]$ but without taking into consideration the condition of volume reflection which accounts for the difference in the soft part of the photon spectrum. However, the results presented in all these papers (for large enough bending radii and not large particle energies) demonstrate the validity of the coherent bremsstrahlung theory in a local area of the bent crystal. Considering the similarity between coherent pair production and bremsstrahlung, the description presented in this paper can be accounted for [15].

The pair photoproduction process in bent single crystals may be used to obtain a linearly polarized high energy $\gamma$ beam and to determine the degree of linear polarization of such beams. In principle, there is the possibility of transforming the linear polarization into a circular one [17,18], but this requires further investigation. The study of this 
field is interesting due to the new opportunity of searching axionlike particles (see for example [19] and literature herein), offered by the unique nature of birefringence for light and high energy photons (the electromagnetic vacuum polarization). Another possible field of application of the photoproduction process in bent single crystals is the cleaning of the gamma part of the halos of future electronpositron colliders. In fact, one may expect the electromagnetic cascade in an oriented bent crystal to be significantly shorter than in an amorphous medium. The use of a multicrystal system could be foreseen for this goal [20].

It should be also noted that the probability and asymmetry functions presented in this paper are smoother and broader in angle for bent crystals compared to straight ones ( $R$ tends to $\infty$ ). For this reason bent crystals are more convenient for the applications described above.

The paper [21] presents also a possible experimental setup to perform the measurements, on the basis of what has already been done for the INSURAD experiment on the CERN SPS extracted beam lines.

\section{ACKNOWLEDGMENTS}

This work was supported by the RFBR Grant No. 08-0201453-a and by the joint Grant No. RFBR 09-02-92431KE-a and EINSTEIN Consortium (Italy). One of the authors (V. A. M) would like to thank the Cariplo Foundation Fellowship for the support and the opportunity for joint work at the Insubria University.

[1] W. Scandale et al., Phys. Rev. Lett. 101, 164801 (2008).

[2] W. Scandale et al., Phys. Rev. A 79, 012903 (2009).
[3] A. G. Afonin et al., JETP Lett. 88, 414 (2008).

[4] M.L. Ter-Mikayelyan, High Energy Electromagnetic Processes in Condensed Media (Wiley, New York, 1972).

[5] V.N. Baier, V.M. Katkov, and V.M. Strakhovenko, Electromagnetic Processes At High Energies In Oriented Single Crystals (World Scientific, Singapore, 1998).

[6] A. R. Mkrtchyan, A. A. Saharian, and V. V. Parazian, Mod. Phys. Lett. B 20, 1617 (2006).

[7] V. A. Maisheev, Phys. Rev. ST Accel. Beams 10, 084701 (2007).

[8] V. A. Maisheev, Phys. Lett. B 477, 83 (2000).

[9] Yu. A. Chesnokov, V. I. Kotov, V. A. Maisheev, and I. A. Yazynin, JINST 3, P02005 (2008).

[10] V. G. Baryshevsky and V. V. Tikhomirov, Sov. Phys. Usp. 32, 1013 (1989).

[11] V. L. Mikhaljov and A. M. Frolov, Yad. Fiz. 41, 962 (1985) (in Russian).

[12] V.A. Maisheev, CERN report, http://indico.cern.ch/ conferenceDisplay.py?confId=50840.

[13] V. A. Arutunov, N. A. Kudryashov, V. M. Samsonov, and M. N. Strikhanov, Nucl. Phys. B363, 283 (1991).

[14] M. V. Bondarenco, arXiv:0908.4503.

[15] Recently, new evidence for this fact has been obtained in the INSURAD test at the H4 beam line with very good agreement between simulation and observations (see [16]).

[16] S. Hasan et al., http://indico.cern.ch/materialDisplay.py? contribId $=843 \&$ sessionId $=58 \&$ materialId $=$ slides $\&$ confId $=73513$.

[17] V. A. Maisheev, arXiv:hep-ex/9904029.

[18] A. Apyan et al. (NA59 Collaboration), Phys. Rev. ST Accel. Beams 11, 041001 (2008).

[19] A. Ringwald, arXiv:1003.2339.

[20] W. Scandale et al., Phys. Rev. Lett. 102, 084801 (2009).

[21] Yu. A. Chesnokov, V.A. Maisheev, D. Bolognini, S. Hasan, M. Prest, and E. Vallazza, arXiv:1006.3391. 